Meta

Journal des traducteurs

Translators' Journal

\title{
Panorama intelectual de la terminología a través del análisis de redes sociales
}

\section{María Rosa Castro-Prieto et María Dolores Olvera Lobo}

Volume 52, numéro 4, décembre 2007

La traduction et les études de réseaux

Translation and Network Studies

URI : https://id.erudit.org/iderudit/017698ar

DOI : https://doi.org/10.7202/017698ar

Aller au sommaire du numéro

Éditeur(s)

Les Presses de l'Université de Montréal

ISSN

0026-0452 (imprimé)

1492-1421 (numérique)

Découvrir la revue

Citer cet article

Castro-Prieto, M. R. \& Olvera Lobo, M. D. (2007). Panorama intelectual de la terminología a través del análisis de redes sociales. Meta, 52(4), 816-838. https://doi.org/10.7202/017698ar
Résumé de l'article

Lorsqu'ils transmettent des connaissances, les auteurs tissent souvent un réseau d'affinités intellectuelles par le biais des travaux qu'ils citent. Ce réseau offre un portrait des grands courants et de l'évolution de leur discipline. Le présent article a pour but de dresser un panorama du domaine de la terminologie en insistant sur ses développements et ses auteurs les plus influents. Pour ce faire, nous nous fondons sur les articles parus dans les périodiques du domaine, sur une période assez longue. L'analyse des citations et la représentation visuelle des relations entre les auteurs par le biais des réseaux sociaux postule que les citations établissent des liens entre les auteurs. Ainsi plus leur fréquence est élevée, plus les affinités entre ces auteurs seront fortes. Notre étude montre par ailleurs que le groupe des auteurs les plus cités représente moins de $1 \%$ du nombre total et que seulement $12 \%$ des auteurs ont publié trois articles ou plus. Enfin, cet article confirme que la terminologie, en tant que champ de recherche, se développe selon trois orientations bien distinctes : les fondements théoriques, le traitement des langues naturelles et la socioterminologie.
Ce document est protégé par la loi sur le droit d'auteur. L'utilisation des services d'Érudit (y compris la reproduction) est assujettie à sa politique d'utilisation que vous pouvez consulter en ligne.

https://apropos.erudit.org/fr/usagers/politique-dutilisation/ 


\title{
Panorama intelectual de la terminología a través del análisis de redes sociales
}

\author{
MARÍA ROSA CASTRO-PRIETO \\ Universidad de Granada, Granada, España \\ mcastro@ugr.es
}

MARÍA DOLORES OLVERA-LOBO

Universidad de Granada, Granada, España

\begin{abstract}
RÉSUMÉ
Lorsqu'ils transmettent des connaissances, les auteurs tissent souvent un réseau d'affinités intellectuelles par le biais des travaux qu'ils citent. Ce réseau offre un portrait des grands courants et de l'évolution de leur discipline. Le présent article a pour but de dresser un panorama du domaine de la terminologie en insistant sur ses développements et ses auteurs les plus influents. Pour ce faire, nous nous fondons sur les articles parus dans les périodiques du domaine, sur une période assez longue. L'analyse des citations et la représentation visuelle des relations entre les auteurs par le biais des réseaux sociaux postule que les citations établissent des liens entre les auteurs. Ainsi plus leur fréquence est élevée, plus les affinités entre ces auteurs seront fortes. Notre étude montre par ailleurs que le groupe des auteurs les plus cités représente moins de $1 \%$ du nombre total et que seulement $12 \%$ des auteurs ont publié trois articles ou plus. Enfin, cet article confirme que la terminologie, en tant que champ de recherche, se développe selon trois orientations bien distinctes: les fondements théoriques, le traitement des langues naturelles et la socioterminologie.
\end{abstract}

\section{ABSTRACT}

When transmitting scientific knowledge, authors weave a web of intellectual affinities through the works they cite that portrays trends and developments in research in their discipline. In the present article, we aim to establish an intellectual panorama of Terminology in which we depict the outstanding developments in research and the most influential authors. To do so, we analyze periodical publications that have appeared over a wide period of time. Author Citation Analysis (ACA) and the visual representation of the relationships between authors through social networks (specifically, pathfinder networks) is based on the premise that links are necessarily established between the authors cited in any specific work so that greater frequency of co-ocurrence indicates a stronger affinity between authors. Among other findings, our results show that the group of most frequently cited authors represents less than $1 \%$ of the total and that only $12 \%$ of authors have published three or more articles. Moreover, we can confirm that research in Terminology is developing in three clearly differentiated directions: theoretical foundations, Natural Language Processing and Socioterminology.

\section{MOTS-CLÉS/KEYWORDS}

social network analysis, cocitation analysis, terminology, scientific production

\section{Introducción}

Aunque la terminología está ligada en sus inicios a las recopilaciones léxicas, no ha sido hasta el siglo XX cuando se ha desarrollado como doctrina con un corpus de 
fundamentos teóricos y metodológicos. Es significativo el hecho de que, aunque esté consensuado como arranque de la materia el año 1931, -en esta fecha se producen dos eventos bibliográficos de gran importancia; por una parte, se publica la tesis titulada Internationale Sprachnormung in der Technik, besonders in der Elektrotechnik: die nationale Sprachnormung und ihre Verallgemeinerung, en una versión ampliada por su propio autor, Wüster, y, por otra, el lingüista ruso Lotte publica el primer artículo sobre esta materia-, su evolución teórica no se haya producido hasta el comienzo de la década de los años setenta. Desde entonces el panorama se ha diversificado de tal manera que se puede hablar de diferentes frentes de investigación y de nuevas propuestas teóricas.

La presente investigación surgió de la necesidad de precisar el panorama intelectual de la terminología mediante un método de trabajo que proporcionara el máximo posible de su conocimiento partiendo de datos objetivos, más allá del conocimiento intuitivo que los especialistas de cada disciplina suelen tener de su materia. De esta manera, se pretende contribuir a mostrar el marco intelectual de la disciplina a través de las publicaciones científicas aparecidas en este ámbito, con la aplicación, además, de técnicas de visualización. Para ello se presta especial atención a los autores, pues no hay que olvidar que son los propios autores los que llevan a cabo las conexiones dentro de la literatura de cada área (Garfield 1994).

En este sentido, White y McCain (1998) presentan una metodología para la identificación de los autores más influyentes de un dominio de especialidad y de las relaciones que mantienen con otros, poniéndose así de manifiesto la estabilidad temática o la evolución de cada autor, además de la posibilidad de reconocer a aquellos que destaquen por representar nuevas corrientes de investigación. El método empleado ofrece una visión cronológicamente delimitada y, por ello, la imagen que se obtiene corresponde, ni más ni menos, al período de tiempo estudiado. Esta característica impide que puedan aparecer frentes demasiado novedosos, que, obviamente, no han podido aún ser plasmados convenientemente.

\section{Material y Métodos}

Para el desarrollo de la investigación se han aplicado una serie de métodos y técnicas desarrollados en los campos de la bibliometría y la sociología, ya suficientemente probados en varias áreas de conocimiento, y que se centran en el análisis de citas de autores (ACA) y en el concepto de redes sociales.

\subsection{Análisis de cocitas}

El recurso a las citas es un instrumento ampliamente reconocido entre los autores. Las referencias que los autores hacen a trabajos previos simbolizan asociaciones conceptuales de ideas científicas reconocidas como útiles por el autor que las cita de manera que los investigadores establecen vínculos entre la investigación en curso y el trabajo previo citado (Garfield 1994). Aunque algunos autores encuentran una correspondencia entre el número de citas recibidas y la calidad del trabajo, otros lo consideran como un indicador de la visibilidad, del uso, de la difusión o del impacto del mismo (González, Moya y Mateos 1997). En cualquier caso, está ya muy asentada la idea de que el recurso a las citas es de gran utilidad en cualquiera de las áreas del 
conocimiento humano, $y$, entre otros efectos, se emplea como una herramienta de evaluación de las actividades de los investigadores.

Desde una perspectiva formal, el autor citado y el autor citante establecen una relación. Desde una perspectiva dinámica, estas relaciones se consideran operaciones relacionales. Pues bien, estas operaciones son recursivas en el sentido de que una cita se refiere a un texto, que, a su vez, contiene otras citas que se refieren a otros textos, y así sucesivamente. De esta forma se genera una red, o una arquitectura, que se extiende a cada momento en el tiempo (Leydersdoff 1998).

Tomando como base el razonamiento anterior, varios investigadores se lanzaron a la tarea de concebir una nueva noción capaz de reflejar analíticamente las interacciones del conocimiento científico. De este modo, surgió el concepto de cocita.

Este concepto fue introducido, de manera paralela, por Small y por Marshakova en el año 1973 y ha sido paulatinamente desarrollado y ampliado por ellos mismos (Small 1997; 1998; Small y Griffith 1974; Small, Sweeney y Greenlee 1985) y por otros autores, como Braam, Moed y van Raan (1991); Chen (1999); Eom (1996); White (1986); White y Griffith (1981, 1982); White y McCain (1998), completando no solo un método de trabajo, sino una técnica ampliamente reconocida y aplicada a diversos campos del conocimiento.

Se define cocita como la frecuencia con la que dos ítems aparecidos en publicaciones anteriores se presentan juntos en una publicación posterior. Ilustramos la definición anterior en la siguiente figura:

FIGURA 1

Esquema de definición de cocita

\begin{tabular}{|c|c|c|}
\hline $\mathrm{A}$ & $\mathrm{C}$ \\
\hline 1998 & & 2002 \\
\hline $\mathrm{B}$ & & $\begin{array}{c}\text { (A 1998 } \\
+\mathrm{B} 2000)\end{array}$ \\
\hline 2000 & \\
\hline
\end{tabular}

En el esquema anterior, A y B representan sendas publicaciones de autores y años diferentes. La flecha indica que ambas se encuentran citadas en una tercera publicación del año 2002. Intuitivamente este hecho permite asociar estos tres documentos, pues en ellos subyace un vínculo que determina la conexión entre los autores y el conocimiento que comparten y transmiten. Gracias a las técnicas desarrolladas por el análisis de cocitas de autores (ACA), esta percepción inicial se ve refrendada, e incluso aumentada, por los resultados obtenidos. El criterio general sobre el que se asienta este concepto se refiere al número de veces que un marcador -cita de autor, de revista o de artículo- ocurre o coocurre con otro, resaltando la información acerca de autores, publicaciones o tendencias de investigación (Ding, Chowdhury y Foo 2001). 


\subsubsection{Análisis de cocitas de autores (ACA)}

White y McCain (1998) parten del concepto "imagen de autor" que se basa tanto en la producción de dicho autor como en la percepción que de él tienen los otros investigadores de su ámbito académico. Dado que en cualquier campo de conocimiento los autores hacen valoraciones -acerca de quién ha escrito qué, usando qué métodos, etc.- que quedan reflejadas mediante sus prácticas citantes, la imagen de un autor se perfila a partir de las citas recibidas realizadas por sus colegas. Sin embargo, solo aquellos autores que reciban un número elevado de citas tienen posibilidad de que esa imagen aparezca reflejada en un mapa generado a partir de las técnicas desarrolladas por el ACA. Esta imagen tiene una doble cara, pues, por un lado, es una imagen fija y, por otro, es una imagen variable. La imagen fija se refiere a la imagen individual de un autor derivada de su producción bibliográfica, que siempre es una imagen constante. En contraste, la imagen variable se refiere a la imagen del autor vinculada a un conjunto cambiante de autores que coocurren citados con él en trabajos de otros investigadores. Para desarrollar adecuadamente la metodología propuesta por este procedimiento, resulta de gran interés tomar la imagen variable de los autores.

El ACA sintetiza las relaciones que se establecen entre los autores que aparecen citados conjuntamente mediante la representación en un mapa en el que cada autor está representado por un punto fijo, que, a su vez, se conecta con el resto de puntos (los autores seleccionados), debido a la repetición de sus coocurrencias. De ello se desprende que autores con perfiles de coocurrencias similares siempre aparecerán juntos. Huelga decir que estas concurrencias muestran una afinidad intelectual entre los autores que no se refiere tanto a la coincidencia de ideas como al interés en el tratamiento de temas similares razón por la cuál son cocitados conjuntamente. Además, si se representaran los mismos autores en más de un período determinado, se estaría en condiciones de observar tendencias y discontinuidades en su trayectoria intelectual: "Such is the history of a field as told by ACA» (White y McCain 1998: 329).

Es evidente que la contribución más sobresaliente que esta técnica reporta a la historia intelectual de la terminología está determinada por la importancia de los autores más citados, por los trabajos que mayor trascendencia han tenido para el desarrollo de la materia -esto es, los trabajos más citados-, por las instituciones más reconocidas en el ámbito e, incluso, por los autores que, pertenecientes a otras disciplinas, también han influido en la materia. En este sentido, Leydersdoff (1998) argumenta que la trama que se crea tiene una doble cara: por un lado, la de las citas con los autores y, por otro, la de las citas con sus publicaciones. De este modo, el recurso a las citas puede funcionar como indicador, tanto de los contextos cognitivos como sociales, de los postulados teóricos del conocimiento; es decir, "citations, as potentially repeated operations, sustain communication in the sciences by drawing upon cognitive and social contexts» (Leydersdoff 1998: 9). Por tanto, la categoría de los datos analizados excede las autorías de los trabajos, puesto que con esta técnica también ofrecen resultados interesantes los trabajos más citados -los que mayor huella dejan en la comunidad científica-, los subcampos que mayor número de contribuciones tienen y las instituciones más significativas de una disciplina de conocimiento (White y McCain 1998). 


\subsection{Análisis de redes sociales}

La organización del conocimiento, su estructura, los modelos de cooperación, el lenguaje y las formas de comunicación reflejan el trabajo de las comunidades científicas y el papel que cumplen en la sociedad. De acuerdo con esto, la mejor manera de comprenderlas implica el estudio de su pensamiento. Se pueden utilizar varios métodos para identificar y describir tipos de patrones de comportamiento o actuación a partir de una matriz de similaridad. Las técnicas de análisis multivariable están ampliamente aceptadas para llevar a cabo este paso previo, tal y como hemos mostrado en publicaciones anteriores (Castro-Prieto 2007) si bien, exigen un conocimiento detallado por parte del investigador para poder interpretarlos adecuadamente. Como alternativa y complemento a estas técnicas, surge el análisis de redes sociales.

El análisis de redes sociales, ha tenido en las últimas décadas un notable interés para las áreas relacionadas con el estudio del comportamiento humano. El concepto de red se establece por el hecho de que cada unidad mantiene vínculos con otras unidades que, a su vez, están vinculadas a otras, y así sucesivamente. Su definición ha sido formalizada por Wasserman y Faust: "A social network consists of a finite set or sets of actors and the relation or relations defined on them" (1994: 20). El análisis de redes permite el estudio de la naturaleza estructural de algunos de los fenómenos sociales y ofrece una nueva plataforma para responder a preguntas irresolutas de las ciencias sociales, pues está en condiciones de ofrecer una definición más precisa de cualquier aspecto de todo tipo de estructuras. De esta forma, el medio social puede ser explicado como un conjunto de pautas regulares en las relaciones entre unidades en interacción. Sin embargo, hay que recalcar que el componente fundamental de las redes sociales no es el individuo, sino la entidad, la cual se define como el conjunto de individuos y conexiones que se establecen entre ellos (Wasserman y Faust 1994).

Las dificultades planteadas por la interrelación entre las unidades se resuelven mediante la aplicación de técnicas estadísticas que aspiran a responder a las preguntas establecidas en la investigación. La interdependencia entre dichas unidades se mide por medio de variables relacionales, que, a su vez, nos abren una amplia gama de posibilidades para analizar los datos y construir modelos que den cuenta de todo tipo de organizaciones, ya sean sociales, de comportamiento, políticas o económicas. La característica distintiva del análisis cuantitativo de redes, frente a otro tipo de análisis de estructuras, reside en que el primero considera como datos definidos matemáticamente las relaciones que se establecen entre las unidades estudiadas.

Por tanto, las relaciones que pueden ser representadas dentro de una comunidad científica son muy diversas. La presente investigación se centra en las relaciones derivadas de la cocitación de autores en las publicaciones periódicas del ámbito de la terminología. Desde el punto de vista metodológico, el análisis de redes sociales, o pathfinder network, consiste en la aplicación de técnicas de visualización del conocimiento las cuáles se han introducido en la metodología del ACA recientemente (Chen, Paul y O’Keefe 2001). Este método es más intuitivo que otros anteriores, y proporciona de una manera más sencilla un paisaje virtual del ámbito de conocimiento analizado. La aplicación de estas técnicas de visualización facilita su comprensión, puesto que utiliza técnicas y algoritmos que contribuyen a "aclarar" la red eliminando los enlaces menos significativos y el resultado es una red simplificada 
que representa la estructura y esencia de la red original. Este aspecto cobra gran importancia en el caso de los investigadores neófitos, al convertirse en una herramienta que puede suministrarles un punto de partida a la imagen intelectual de la disciplina a la que se aproximan o, al menos, un marco de referencia que les permita un acercamiento sencillo a la misma. La evolución de los nuevos modelos y tecnologías de visualización del conocimiento mejoran notablemente la aproximación tradicional al estudio de las disciplinas científicas (Chen, Paul y O’Keefe 2001).

\subsubsection{Medidas de centralidad}

Para la correcta visión que del mapa de la terminología se va a hacer a continuación, conviene explicar que este se representa mediante un conjunto de puntos y líneas que, respectivamente, simbolizan a los autores más citados de la materia terminológica, así como las relaciones que mantienen entre ellos - determinadas por las veces que coocurren juntos-. Además de los vértices o puntos que simbolizan las unidades, en este caso personificados por los autores objeto de este estudio, las líneas configuran uno de los elementos imprescindibles de la representación gráfica de redes, pues, al convertir los datos de la matriz en un gráfico, se representan las relaciones por medio de ellas. Luego se puede afirmar que las líneas representan el prototipo elemental de las conexiones entre los vértices, convirtiéndose éstos últimos en el fundamento del análisis y constituyendo la estructura social del sistema.

Existen diferentes valores que cuantifican la relevancia que tiene un nodo, dentro de una red, frente al resto de nodos. Estos valores se refieren al concepto de centralidad, cuyas soluciones explican, mediante los vínculos, las entidades "más visibles", en contraste con las "más aisladas" (Wasserman y Faust 1994). El concepto de centralidad revela que cuanto mayor sea el grado de implicación de una entidad dentro de una estructura, mayor será el número de relaciones que establezca en relación a los demás. Las medidas de centralidad son tres: grado, cercanía y nivel de intermediación.

a) Grado: Se suele afirmar que la centralidad de un punto está en función del grado de ese punto. El grado de un punto tiene que ver con el número de puntos que son adyacentes a él, y con los que está, por tanto, en contacto directo. Se acepta que un autor que ocupa una posición que le permita estar en contacto directo con muchos otros es un importante canal de información y, por consiguiente, también lo serán sus contribuciones. Chen (Chen, C.; Paul, R. y O’Keefe, B.; 2001) reconoce como fundamental entender la comunicación científica a través del estudio de dominios del conocimiento como comunidades de discurso. La visualización de un dominio abre muchas oportunidades de explorar los cambios dentro del mismo y en varios niveles. Estos mapas resultantes permiten seguir los enlaces que se originan en un autor de una disciplina y a través de la citación, representada en los enlaces, examinar las relaciones con otros autores y trabajos.

b) Cercanía: La ubicación central garantiza una mejor comunicación de la información, pues ocupar esta situación significa que ese punto interactúa con más entidades. Entra en juego, entonces, la medida de cercanía, en la que el concepto de distancia cobra gran relevancia. En efecto, cuanto menor sea el trayecto entre los nodos, tanto mayor será la relación entre ellos. De nuevo, el autor que ocupe el lugar central de la red, además de que su distancia será mínima con respecto a los demás, también será un aval para que la información circule en el menor tiempo posible. Freeman (2000) explica que las distancias cortas significan menos intermediarios, menos tiempo de transmisión de la 
información y más eficiencia en la comunicación, puesto que, al tener un mayor nivel de cercanía con los restantes autores de la red, se asegura un mayor flujo de la información.

c) Elementos intermediarios: La tercera medida utilizada para medir la prominencia de los nodos de una red recibe el nombre de elemento intermediario (betweenness). Esta medida, al igual que el grado, está relacionada con la idea de conexión, puesto que, si hay dos nodos adyacentes conectados por un tercero, desde el momento en que el tercer nodo "desaparece" se pierde esa conexión. Es decir, cesa el flujo de información entre esas dos unidades, lo que, extrapolado a los resultados que se arrojan en el análisis de citas, es lo mismo que decir que esos autores se han desconectado del resto. De este modo, esta medida subraya el poder que tienen determinados nodos de conectar recursos que de otra forma quedarían desconectados.

Para la realización del presente trabajo se parte de la asociación de los dos procedimientos comentados anteriormente (ACA y redes sociales). La construcción de un engranaje social que dé cuenta de los lazos que unen a los autores con sus homónimos citados constituye el trasfondo sobre el cual se asienta la estructura de la materia de conocimiento que fundamenta este trabajo: la terminología.

\subsection{Tratamiento y selección de los datos}

En varios estudios realizados con ACA para otros ámbitos de conocimiento las publicaciones analizadas se han referido principalmente a las incluidas en la base de datos de índices de citas del ISI (actualmente registrado como Thomson Scientific). Como para este estudio únicamente es la revista Meta, Le Journal du Traducteur la que está incluida en $A \& H C I^{\circ}$ (Arts \& Humanities Citation Index) hubo que seleccionar las publicaciones que iban a analizarse aplicando otros criterios. El análisis de la estructura intelectual de la terminología se llevó a cabo a partir de los artículos relacionados con esta disciplina incluidos en publicaciones periódicas especializadas, si bien se utilizaron una serie de parámetros con el fin de acotar el corpus de documentos temática, cronológica e idiomáticamente (Castro-Prieto y Olvera-Lobo 2003a; Castro-Prieto y Olvera-Lobo 2003b). Se tuvieron en cuenta las revistas científicas, puesto que concentran el mayor número de estudios en este campo, y no se tomaron en consideración aquellas publicaciones de periodicidad anual, tales como actas de seminarios y reuniones o boletines informativos.

Los datos bibliográficos extraídos de estas publicaciones se volcaron a una base de datos diseñada y creada ad hoc, con el fin de facilitar la introducción, gestión y recuperación de la información, así como la aplicación de los métodos cuantitativos que permitiesen desarrollar los procesos de análisis (Castro-Prieto y Olvera-Lobo 2003a).

\section{3.a. Delimitación temática}

La finalidad principal del trabajo consistió en conocer la situación actual, las nuevas corrientes de investigación y la relación de la disciplina terminológica con otras afines a partir de los artículos publicados en revistas especializadas, fuente fundamental para establecer la preeminencia de ideas y la influencia de unos autores sobre otros. La tabla 1 muestra las revistas utilizadas en nuestro estudio. 
TABLA 1

Relación de revistas estudiadas

\begin{tabular}{||l|l||}
\hline Revistas de terminología & Revistas de campos afines \\
\hline - La banque des mots & - Meta, Le Journal du Traducteur \\
\hline - Lsp \& Professional Communication & - Terminology \& Translation \\
\hline - Terminologies Nouvelles & - Traduction, Terminologie, Redaction \\
\hline - Terminology International Journal of \\
$\begin{array}{l}\text { Theoretical \& Applied Issues in } \\
\text { Specialized Communication }\end{array}$ & \\
\hline - Terminology, Science \& Research & \\
\hline - Unesco Alsed-Lsp Newsletter & \\
\hline
\end{tabular}

Si bien todas las revistas integrantes del presente estudio son reconocidas por los expertos como pertenecientes a la disciplina, se distinguen aquellas cuyo tema predominante es la terminología en todos sus aspectos y las que dedican uno de sus apartados en exclusiva a la terminología, pero sin descuidar otros campos afines como la traducción o la redacción técnica.

\section{3.b. Delimitación cronológica}

Se tomó como punto de partida cronológico del estudio el inicio de cada una de las publicaciones tratadas, con el fin de ser más exhaustivos en la investigación.

Aunque se puede considerar la década de los ochenta como la fecha en la que la terminología, por sí misma, empieza a perfilarse como objeto de reflexión teórica de una manera regular, la aparición de sus primeras publicaciones periódicas se inicia en 1967 con la revista Méta. No obstante, el incremento de la producción científica que se produce desde el final de los años 70, tal y como lo demuestra la aparición en el mercado editorial de las primeras obras de corte exclusivamente teórico -Dubuc, 1978 Manuel pratique de terminologie; Sager, Dungworth y McDonald, 1980 English Special Languages: Principles and Practice in Science and Technology; Wüster, 1979 Einfürung in Die Allgemeine Terminologielhere Und Terminologische Lexicographie -, marca lo que se puede considerar como el inicio del pensamiento sistemático sobre esta materia. A esto hay que añadir la profusión de encuentros, simposios, jornadas y congresos en torno a este campo durante esta década, si bien hay que remontarse hasta la segunda mitad de los años setenta para certificar el origen de las primeras reuniones de esta índole.

Hemos fijado como fecha de cierre de toma de los datos el año 2001, correspondiente al comienzo de esta investigación, tal y como recomiendan White y McCain (1998), además de cerrar un ciclo temporal extenso, pues consideramos que treinta y cinco años de publicaciones terminológicas son suficientes para avalar el contenido de este estudio.

\section{3.c. Delimitación idiomática}

La delimitación idiomática que hemos impuesto en la recogida de los datos evidencia que son el francés y el inglés las lenguas que más documentos escritos concentran en esta disciplina: los autores más relevantes de esta área de conocimiento, aunque sean hablantes de otras lenguas, utilizan con frecuencia alguna de estas dos para transmitir 
a la comunidad científica sus hallazgos. Aunque en un primer momento no se contempló la posibilidad de incluir publicaciones ni en lengua española ni en lengua alemana, la manipulación y el posterior análisis de los datos nos ha convencido de su conveniencia para lograr una mejora y ampliación de la información puesto que esto contribuiría a una mayor exhaustividad de las producción analizada.

\subsection{Localización de los datos}

Con el fin de recopilar la información bibliográfica de los artículos científicos referidos al campo de la terminología y de conocer la ubicación física de las revistas para cotejar y recoger todos los datos necesarios, se consultaron catálogos automatizados de bibliotecas universitarias; índices de citas, en este caso el SSCI (Social Sciences Citation Index) y el AઐHCI (Arts \& Humanities Citation Index); el directorio internacional de publicaciones periódicas Ulrich's On Disc de Bowker, así como la base de datos bibliográfica MLA (Modern Language Association of America), que recoge publicaciones del área de la lingüística.

Como no toda la información bibliográfica estaba automatizada, hubo que cargar datos de forma manual. Debido a la falta de normalización por parte de las publicaciones, fue necesaria una homogeneización de los campos directamente relacionados con el objeto de estudio (autores, instituciones, revistas, descriptores) para su identificación y posterior análisis. La tabla 2 resume el contenido de los campos cuantificables de la base de datos creada para la presente investigación (Castro-Prieto 2007: 140-148; Castro-Prieto y Olvera-Lobo 2003b). Además, se incluyen otros campos como título del artículo, volumen de la revista o filiación del autor.

TABLA 2

La base de datos en cifras

\begin{tabular}{|c|c|c|c|c|c|c||}
\hline Artículos & Revistas & $\begin{array}{c}\text { Autor(es) de } \\
\text { los artículos }\end{array}$ & $\begin{array}{c}\text { Años de } \\
\text { publicación } \\
\text { de las } \\
\text { revistas }\end{array}$ & $\begin{array}{c}\text { Autores } \\
\text { citados }\end{array}$ & $\begin{array}{c}\text { Años de } \\
\text { publicación } \\
\text { de los } \\
\text { trabajos } \\
\text { citados }\end{array}$ & Descriptores \\
\hline 1072 & 9 & 750 & $1967-2001$ & 4369 & s.XVI-s.XX & 20 \\
\hline \hline
\end{tabular}

\section{Resultados: Análisis de los datos}

El análisis de la información bibliográfica recogida ha proporcionado una serie de datos cuantitativos acerca de la producción científica de la terminología. Se han obtenido unos indicadores que ayudan a conocer cómo ha evolucionado el flujo de conocimiento de dicha disciplina durante el período temporal estudiado. Para ello se han estudiado aspectos relativos a la producción (productividad en revistas) y al consumo de información (lo que se refleja a partir de las citas recibidas por los autores). Para un mayor detalle de la productividad de los autores o de las instituciones, así como de la producción de citas por año, o por año y revista, o de los artículos y citas por año, pueden consultarse los trabajos de Castro-Prieto (2007); Castro-Prieto y Olvera-Lobo (2003a); Castro-Prieto y Olvera-Lobo (2003b). 


\subsection{Análisis de la producción científica}

Algunos de los indicadores utilizados para analizar la evolución de la terminología como disciplina científica se refieren a la producción de artículos (gráfico A) y la producción de citas (tabla 3) en revistas especializadas. En el gráfico A se representa el incremento de la producción de artículos desde el principio de los años noventa, destacando hacia mediados de la misma década un aumento considerable, cercano a los cien artículos en algunos momentos, aunque sin superar esta cifra. Son varias las circunstancias que revelan la razón de este crecimiento tan notable: (a) la aparición de nuevas revistas, (b) la consagración de números monográficos a cuestiones teóricas y metodológicas de la terminología y (c) la proliferación de la publicación en revistas de las contribuciones en congresos. Todo esto repercute en un aumento de la producción de citas en los trabajos, tal y como veremos más adelante. El paralelismo, pues, es evidente, ya que el aumento de citas se encuentra indiscutiblemente ligado a este fenómeno.

GRÁFICO A

Progresión lineal de artículos por año

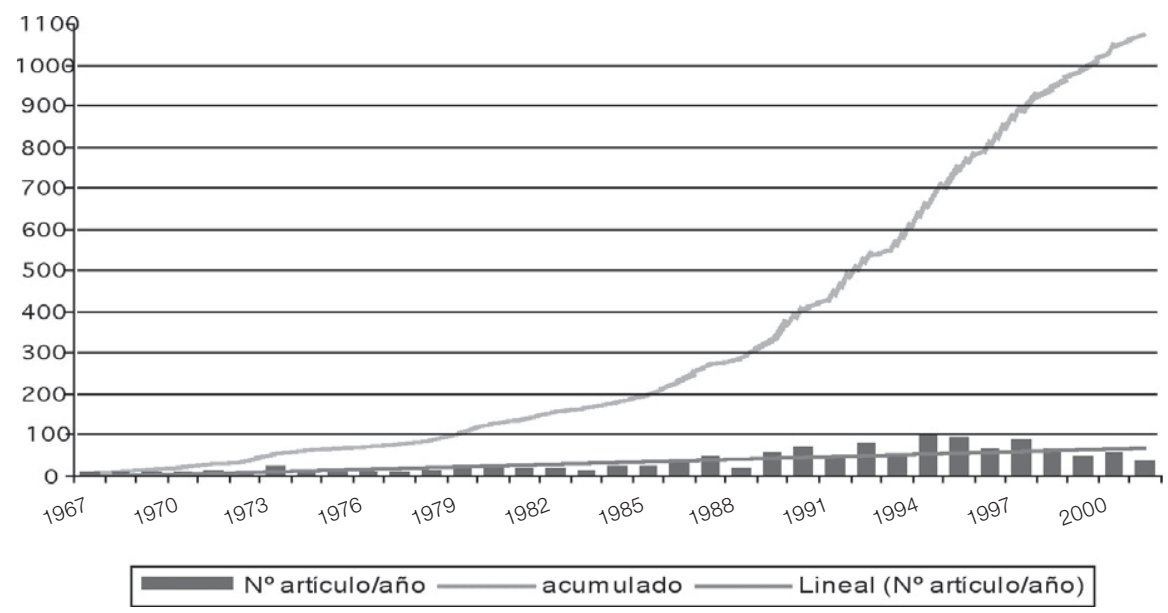

Si bien a priori puede parecer que el mayor número de artículos garantiza una mayor producción de citas, varios casos contradicen este supuesto. En primer lugar, fijémonos en los casos de La banque des mots y de Terminologie \& Traduction, que, a pesar de tener más de cien artículos cada una de ellas, no llegan a las mil citas; es decir, su elevada producción científica no se corresponde con su volumen de citas. En el caso opuesto, Terminology International Journal muestra una elevada producción de consumo de la información que no se corresponde con su producción de artículos, debido a la juventud de la revista estudiada, lo cual hace pensar que, si sigue con esta tendencia, puede liderar la productividad, habida cuenta de que sus contenidos se dedican con exclusividad a los estudios terminológicos.

Es oportuno cuantificar cuáles son los autores que reciben un mayor volumen de citas, así como cuál es la mayor producción de las mismas desde el punto de vista de las revistas, antes de considerar la influencia y conexiones entre ellos. Desde la 
perspectiva de la distribución del volumen de citas recibidas, sobresale un número muy escaso de autores (4369) en comparación con la cifra total de receptores de las mismas. En concreto, los más citados, es decir, los citados más de cincuenta veces, son quince, cuyo volumen oscila entre este mínimo y un máximo de ciento cuarenta y una citas. El resto no alcanzan las veinte citas. Los trabajos de estos autores, publicados en las décadas de los años ochenta y noventa del pasado siglo, son los que mayor número de veces han sido citados en comparación a los trabajos publicados en décadas anteriores.

En la tabla 3 se expone la nómina de los autores que han recibido más de 50 citas - una vez eliminadas todas sus autocitas -, así como el número máximo de citas recibidas por el resto de autores hasta un mínimo de cinco.

TABLA 3

Total de citas recibidas por autor

\begin{tabular}{|l|l||}
\hline Autores citados & Número de citas \\
\hline SAGER, J.C. & Hasta 150 citas \\
\hline $\begin{array}{l}\text { LERAT, P. } \\
\text { REY, A. }\end{array}$ & Hasta 100 citas \\
\hline $\begin{array}{l}\text { FELBER, H. } \\
\text { GAUDIN, F. } \\
\text { PICHT, H. } \\
\text { WUSTER, E. }\end{array}$ & Hasta 90 citas \\
\hline $\begin{array}{l}\text { GUILBERT, } \text {. } \\
\text { KOCOUREK, } \text { R. }\end{array}$ & Hasta 80 citas \\
\hline $\begin{array}{l}\text { AUGER, P. } \\
\text { CABRÉ, M. T. } \\
\text { MEYER, I. }\end{array}$ & Hasta 70 citas \\
\hline $\begin{array}{l}\text { BOULANGER, J.C. } \\
\text { BOURIGAULT, D. } \\
\text { KAGEURA, K. }\end{array}$ & Hasta 60 citas \\
\hline $\begin{array}{l}\text { DUBUC, R. } \\
\text { HEID, U. } \\
\text { MEL'CUK, I. } \\
\text { RONDEAU, G. }\end{array}$ & Hasta 50 citas \\
\hline 15 autores & Hasta 40 citas \\
\hline 26 autores & Hasta 30 citas \\
\hline 89 autores & Hasta 20 citas \\
\hline 192 autores & Hasta 10 citas \\
\hline 4028 autores & Hasta 5 citas \\
\hline \hline
\end{tabular}

Los resultados de estas operaciones han demostrado que, por un lado, J.C. Sager es el autor más veces citado, con una amplia ventaja sobre el resto de autores, y, por otro lado, que el conjunto de autores que mayor cantidad de citas acumulan apenas alcanza el $1 \%$ del total.

Se observa que el grupo de los menos citados -aquellos que han recibido cuarenta o menos de cuarenta citas-, además de ser el bloque más numeroso, contiene un buen número de investigadores que no pertenecen al campo de la terminología, aunque sus trabajos hayan sido importantes para la disciplina (Castro-Prieto 2007). En efecto, 
la mayoría de ellos han desarrollado sus intereses investigadores en el campo de la lingüística, iniciando nuevas vías de pensamiento, pero sus innovaciones han trascendido su propia disciplina. Aunque con algunas precisiones, se puede afirmar que casi todos los autores han relacionado sus intereses investigadores con la disciplina terminológica. Pertenecen a este conjunto nombres tan representativos de la disciplina como Sager, Wüster, Gaudin o Cabré, que han iniciado corrientes de pensamiento determinantes para la evolución de la materia.

En el gráfico B se ha representado el volumen de citas por año, independientemente de la revista productora, mediante un diagrama de barras. Es notable que el volumen de citas sea bastante discreto hasta mediados de los noventa. Sin embargo, tal y como hemos apuntado anteriormente, esta tendencia cambia radicalmente a partir del año 1993, en el que se pasa de una producción de cuatrocientas cincuenta y cuatro citas a una de mil doscientas setenta y seis al año siguiente. Si se echa una rápida ojeada a la fuente de datos, se distingue una clara diferencia entre los contenidos, avalados por los descriptores, de los artículos escritos en fechas anteriores a la señalada, y comprobamos que en los de fechas posteriores se observa una mayor cantidad de escritos teóricos. A este cambio tan espectacular también hay que añadir el incremento de la producción de artículos ya comentado.

Merece un comentario aparte el hecho de que desde que se inicia el recuento de artículos y citas de la disciplina, y hasta 1978, los únicos artículos recopilados corresponden a la revista Meta, puesto que es la única que publica trabajos de terminología durante ese período. Sin embargo, se debe señalar que la ausencia de citas en las publicaciones terminológicas es absoluta hasta el año 1973, tal y como se observa en el gráfico B. Por otra parte, hay que destacar que, a partir de este momento -si se exceptúa el año 1998-, se mantiene una cierta estabilidad en la progresión entre el número de artículos y sus respectivas citas.

GRÁFICO B

Productividad de citas por año

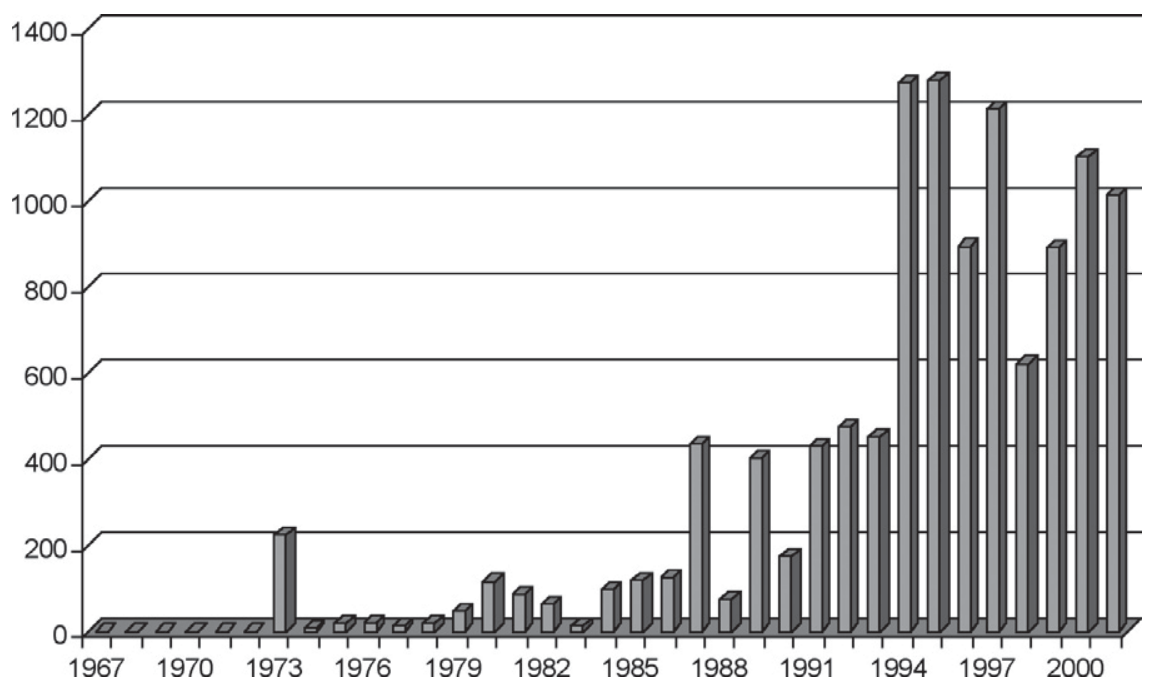


Se constata el crecimiento constante que se ha producido desde el comienzo de la década de los noventa. Hasta este año se habían acumulado, desde el inicio de la producción, mil ochocientas setenta y siete citas y, a partir de este momento, en una progresión continua, se incrementa de tal forma que en 2001 se llega a las once mil setecientas once.

\subsection{Aplicación de nuevas técnicas}

Una vez presentados los resultados directamente observables de la base de datos en el epígrafe anterior, aquí se mostrarán los datos que se infieren de su contenido.

Las pautas de actuación de autores y publicaciones se han detallado en dos planos que permitirán realizar el análisis global de la investigación llevada a cabo en el campo de la terminología (Castro-Prieto y Olvera-Lobo 2003a; 2003b). Mientras que el plano anterior se corresponde con un análisis descriptivo de documentos y se orienta a la cuantificación de la evolución productiva de la especialidad por medio de las revistas, los trabajos, los autores o los tipos de instituciones productoras, el segundo, de corte sociológico y con un carácter experimental, corresponde a un análisis de los autores y, por ende, de su conocimiento. Se examina la estructura intelectual de la disciplina terminológica, las redes científicas, en un periodo temporal que abarca unos treinta y cinco años aproximadamente. El análisis e interpretación de estos datos permitirá mostrar un panorama general sobre el estado actual de la disciplina.

\subsubsection{Análisis de cocitas de autores (ACA)}

La secuencia seguida para el análisis de los datos se fundamenta en la propuesta realizada por White (1986) y McCain (1990) ya expuesta en el epígrafe 2 . Una vez identificados los elementos que se sometieron al análisis de las diferentes técnicas -en este caso sesenta autores que obtuvieron más de veinte citas y que coocurrían en publicaciones periódicas de terminología (tabla 4)-, se determinó el número de veces que cada par había sido citado conjuntamente y se creó una matriz de cocitas que mostraba las relaciones entre ellos. En la siguiente etapa la matriz derivó en una matriz de correlaciones que permitió la conversión de las frecuencias de cocitas en coeficientes de correlación. En la última fase del procedimiento se representaron las relaciones que se establecen entre los autores mediante el análisis de redes sociales, a través del cual se observan los autores más prominentes e influyentes de la disciplina.

TABLA 4

Número de citas de los autores más citados

\begin{tabular}{||l|c|l|c||}
\hline Autores más citados & Número citas & Autores más citados & Número citas \\
\hline SAGER, J.C. & 141 & PAVEL, S. & 33 \\
\hline REY, A. & 93 & AHMAD, K. & 32 \\
\hline LERAT, P. & 92 & BUDIN, G. & 32 \\
\hline PICHT, H. & 89 & SCHAETZEN, C. & 31 \\
\hline FELBER, H. & 88 & THOIRON, P. & 29 \\
\hline GAUDIN, F. & 85 & ARNTZ, R. & 28 \\
\hline
\end{tabular}




\begin{tabular}{|c|c|c|c|}
\hline WUSTER, E. & 83 & DUNGWORTH, D. & 27 \\
\hline KOCOUREK, R. & 79 & GOUADEC, D. & 27 \\
\hline GUILBERT, L. & 75 & HALLIDAY, M. A. K. & 27 \\
\hline AUGER, P. & 63 & CHURCH, K. W. & 26 \\
\hline CABRE, M. T. & 63 & GALINSKI, C. & 26 \\
\hline MEYER, I. & 60 & MACKINTOSH, K. & 26 \\
\hline BOULANGER, J.C. & 59 & REY-DEBOVE, J. & 26 \\
\hline KAGEURA, K. & 56 & CONDAMINES, A. & 25 \\
\hline BOURIGAULT, D. & 52 & LYONS, J. & 25 \\
\hline MEL'CUK, I. & 46 & MCDONALD, P. F. & 25 \\
\hline DUBUC, R. & 44 & ROUSSEAU, L. J. & 25 \\
\hline HEID, U. & 44 & BEAUGRANDE, R. & 24 \\
\hline RONDEAU, G. & 43 & CORBEIL, J.C. & 24 \\
\hline CLAS, A. & 38 & BEJOINT, H. & 23 \\
\hline KLEIBER, G. & 38 & DAHLBERG, I. & 23 \\
\hline ANANIADOU, S. & 37 & DRASKAU, J. & 23 \\
\hline GAMBIER, Y. & 36 & DUBOIS, J. & 23 \\
\hline QUEMADA, B. & 36 & SINCLAIR, J. & 23 \\
\hline JACQUEMIN, C. & 35 & LAKOFF, G. & 22 \\
\hline HUMBLEY, J. & 34 & OLF & 21 \\
\hline OTMAN, G. & 34 & SLODZIAN, M. & 21 \\
\hline RASTIER, F. & 34 & MORTUREUX, M. F. & 20 \\
\hline DAILLE, B. & 33 & ROGERS, M. & 20 \\
\hline GUESPIN, L. & 33 & TSUJII, J. & 20 \\
\hline
\end{tabular}

De una lista inicial de once mil setecientos once ítems (11711) correspondientes al número de citas incluidas en los artículos estudiados, se ordenaron los autores y se contabilizó el número de veces que eran citados, tras lo que se procedió a normalizar el nombre de los autores. La cifra de autores diferentes citados ascendió a cuatro mil trescientos sesenta y nueve (4369 autores). Se asignó el número de citas a cada autor y se eliminaron los que tenían diecinueve citas o menos. Conviene aclarar que se ha desechado de esta segunda lista las citas que los autores hacen de sus propios trabajos, es decir, las autocitas, con lo que queda un resultado de sesenta autores (tabla 4). Por limitaciones de software, la cifra final de autores con los que se iba a trabajar no podía superar la centena.

El siguiente paso consistió en convertir los datos en una matriz de correlación de las citas de los sesenta autores más citados. El objetivo de esta operación era conseguir que los datos "en bruto" se convirtieran en valores que mostraran la frecuencia con la que los autores coocurrían entre sí. No obstante, los datos incluidos en la matriz, por sí solos, no revelan ningún tipo de resultados. Se aplicó un coeficiente de correlación -en este caso Pearson- para determinar el grado de relación entre cada par de datos. Aunque los autores incluidos en la matriz contaban con un mínimo de veinte citas, la frecuencia de aparición "por pares" fue muy variada. La mayor parte de las celdas estaban a cero, es decir, esos autores no coocurrían jamás, y los frecuentes casos en los que coocurrían una o dos veces no resultaban demasiado significativos, pues no aportaban información relevante para la investigación en curso. En contraposición, 
aquellos cuyas celdas tenían una cifra elevada coocurrían tantas veces y en tantos trabajos como la cifra indicaba. Por lo tanto, la metodología empleada permite inferir que aquellos pares de autores que presentan un valor elevado de concurrencias forman parte de una misma tendencia o, al menos, comparten similares intereses de investigación. Es decir, que determinados investigadores que publican trabajos sobre un tema concreto, han hecho referencia conjunta a esos autores. Del mismo modo, permite deducir lo contrario, es decir, que los pares con una frecuencia nula, o baja, de coocurrencia no son afines intelectualmente.

\subsection{Aplicación de la red social}

Para generar las representaciones de redes sociales, se ha utilizado el software que Batagelj y Mrvar (1996) desarrollaron para este fin, denominado Pajek.

Para la correcta visión que del mapa de la terminología se va a hacer a continuación, conviene explicar que este se representa mediante un conjunto de puntos y líneas que, respectivamente, simbolizan a los autores más citados de la materia terminológica, así como las relaciones que mantienen entre ellos -determinadas por las veces que coocurren juntos-. Además de los vértices o puntos que simbolizan las unidades, en este caso personificados por los autores objeto de este estudio, las líneas configuran uno de los elementos imprescindibles de la representación gráfica de redes, pues, al convertir los datos de la matriz en un gráfico, se representan las relaciones por medio de ellas. Luego se puede afirmar que las líneas representan el prototipo elemental de las conexiones entre los vértices, convirtiéndose éstos últimos en el fundamento del análisis y constituyendo la estructura social del sistema.

Como se explicó en el epígrafe 2, existen diferentes valores que cuantifican la relevancia que tiene un nodo, dentro de una red, frente al resto de nodos. Estos valores se refieren al concepto de centralidad, cuyas soluciones explican, mediante los vínculos, las entidades "más visibles", en contraste con las "más aisladas" (Wasserman y Faust 1994). El concepto de centralidad revela que cuanto mayor sea el grado de implicación de una entidad dentro de una estructura, mayor será el número de relaciones que establezca en relación a los demás. Al hilo de esta afirmación, y relacionándola con los resultados del presente trabajo, estamos en condiciones de atestiguar que los autores que se encuentran en el centro de la red, que representa la estructura de la disciplina terminológica, constituyen el referente principal de las contribuciones más significativas al área de conocimiento en cuestión, además de ser los más "reconocidos", mientras que los autores localizados en su periferia, al tener menos conexiones, quedan en una posición de menor importancia. Es decir, la posición centro o periferia viene dada por el número de relaciones ya que el software adjudica una posición más central a los autores más conexionados para facilitar la visualización de los enlaces colocando en la periferia a los que menos vínculos presentan.

El mapa resultante (gráfico C) se interpretará mediante la aplicación de las medidas de centralidad - grado, cercanía, elementos intermediarios- a la ubicación que ocupan los puntos en la red, cuyo objetivo inmediato es el de clarificarlos al especificar sus componentes y las relaciones que mantienen entre ellos (Freeman 2000). 


\section{a) Grado}

Tal y como se puede comprobar en la tabla 5, el autor que tiene el máximo grado posible de conexiones es Sager, pues tiene grado treinta y dos (32), lo cual permite atestiguar que es el "foco" más importante de comunicación. Obviamente, el resto de autores que le acompañan en la tabla son considerados del mismo modo. A medida que los autores se alejan del centro, su grado pierde intensidad. De hecho, los autores ubicados en la parte externa no suelen tener más de un grado 5 -Tsujii, Ananiadou, Rogers, Church, Sinclair, Schaetzen, Rousseau, entre otros-, por lo que se considera que tienen un grado bajo de conexiones. Freeman (2000) indica que la posición que ocupan estos puntos los aísla de una relación directa con la mayoría de los otros y los separa de una participación activa en el proceso de comunicación.

TABLA 5

Nómina de autores con grado mayor de 15

\begin{tabular}{||l|c|l|c||}
\hline Autores & Grado & Autores & Grado \\
\hline SAGER & 32 & REY & 19 \\
\hline CABRE & 27 & GAUDIN & 18 \\
\hline PICHT & 22 & AHMAD & 16 \\
\hline LERAT & 22 & KOCOUREK & 16 \\
\hline FELBER & 19 & AUGER & 15 \\
\hline
\end{tabular}

b) Cercanía

En el gráfico C se constata lo expuesto anteriormente. En efecto, Sager, Wüster y Picht son los autores cuya situación en la red, indiscutiblemente, es la más céntrica e, incluso, si aumentamos algo más el diámetro del círculo central incluiríamos a Cabré, Ahmad y Felber, que pasarían a ser autores cuya posición garantiza la fluidez de la información. Se constata que, si exceptuamos a Wüster, los demás autores tienen un alto grado de conexiones (véase tabla 5). Aclaramos que, después del recuento de vínculos, Wüster obtuvo trece ligazones, por lo que no fue incluido en la tabla de los autores con mayor grado.

\section{c) Elementos intermediarios}

En el gráfico D se representan los autores que mayor índice de intermediación tienen mediante un punto de mayor tamaño. Auger, Cabré, Felber, Lerat, Picht y Sager destacan como nodos más importantes, pues recordemos que su grado, es decir, la suma de sus conexiones, está entre los mayores de la red (véase tabla 5), lo que significa que, si "desaparecen", quedan desconectados tantos autores como número de conexiones mantienen. En el caso opuesto se sitúan aquellos autores que al tener un bajo grado de conexiones, además de encontrarse en la periferia de la red, no suponen pérdida de información, al no desconectar ningún nodo $y$, por lo tanto, no tienen ningún efecto sobre las relaciones de los demás.

Si se comparan las soluciones dadas de la aplicación de las medidas de grado, cercanía e intermediación, no sorprende que Cabré, Felber, Picht y Sager sean los autores que indican mayor grado de centralidad en la red y, como consecuencia, sean los autores más prominentes en terminología, ya que son los que concentran un 
mayor número de conexiones con el resto de los autores, tienen un mayor número de distancias geodésicas y, por tanto, aseguran el mayor flujo de información de la disciplina.

En el gráfico E se ha representado las conexiones de semejanza -entendida, en este caso, como afinidad intelectual- que unen a los autores. Estas líneas se han obtenido en función del número de veces que los autores aparecen citados conjuntamente. Así, en el gráfico E se exponen únicamente aquellos autores cuyo vínculo es más intenso, de lo que se desprende que estos forman "familias de conocimiento" o, lo que es lo mismo, que corresponden a los mismos frentes de investigación.

A partir de la matriz de frecuencia de cocitas, el programa Pajek (Batagelj y Mrvar 1996) ha establecido las ligazones que con mayor fuerza "atan" a los autores. Destaca la conexión que une a Wüster con su discípulo Felber, pues, de todas, es la más fuerte. A su vez, ambos están conectados a Sager, aunque su unión va perdiendo intensidad de Felber a Sager y de este a Wüster. Se observan más relaciones tripartitas, aunque no todas de la misma intensidad, dentro de la estructura de la red. De entre todas, destacan las que unen a Gaudin, Gambier y Guespin -representantes de la corriente denominada socioterminología-, a Daille, Jacquemin y Bourigault -pertenecientes al frente de investigación que hemos denominado procesamiento del lenguaje natural-, y también las que unen a Budin, Ahmad y Picht, o a Arntz, Picht y Wüster -correspondientes a la corriente que dio origen a la disciplina, es decir, a la teoría general de la terminología-. La situación que ocupan los autores en el mapa estructurado de la terminología indica las tendencias de investigación más definidas dentro de la disciplina. Estas tendencias coinciden con las ya apuntadas en publicaciones anteriores obtenidas de los resultados de la aplicación de las técnicas de análisis multivariante (Castro-Prieto 2007).

Como se puede observar, los nodos situados en el centro, o lo que es lo mismo, Picht, Sager y Wüster, son los que más conexiones tripartitas establecen (véase gráfico E). Esto quiere decir que son los que comparten mayor semejanza intelectual con aquellos con los que forman un triángulo. 
GRÁFICO 1

Representación de la red social de la terminología

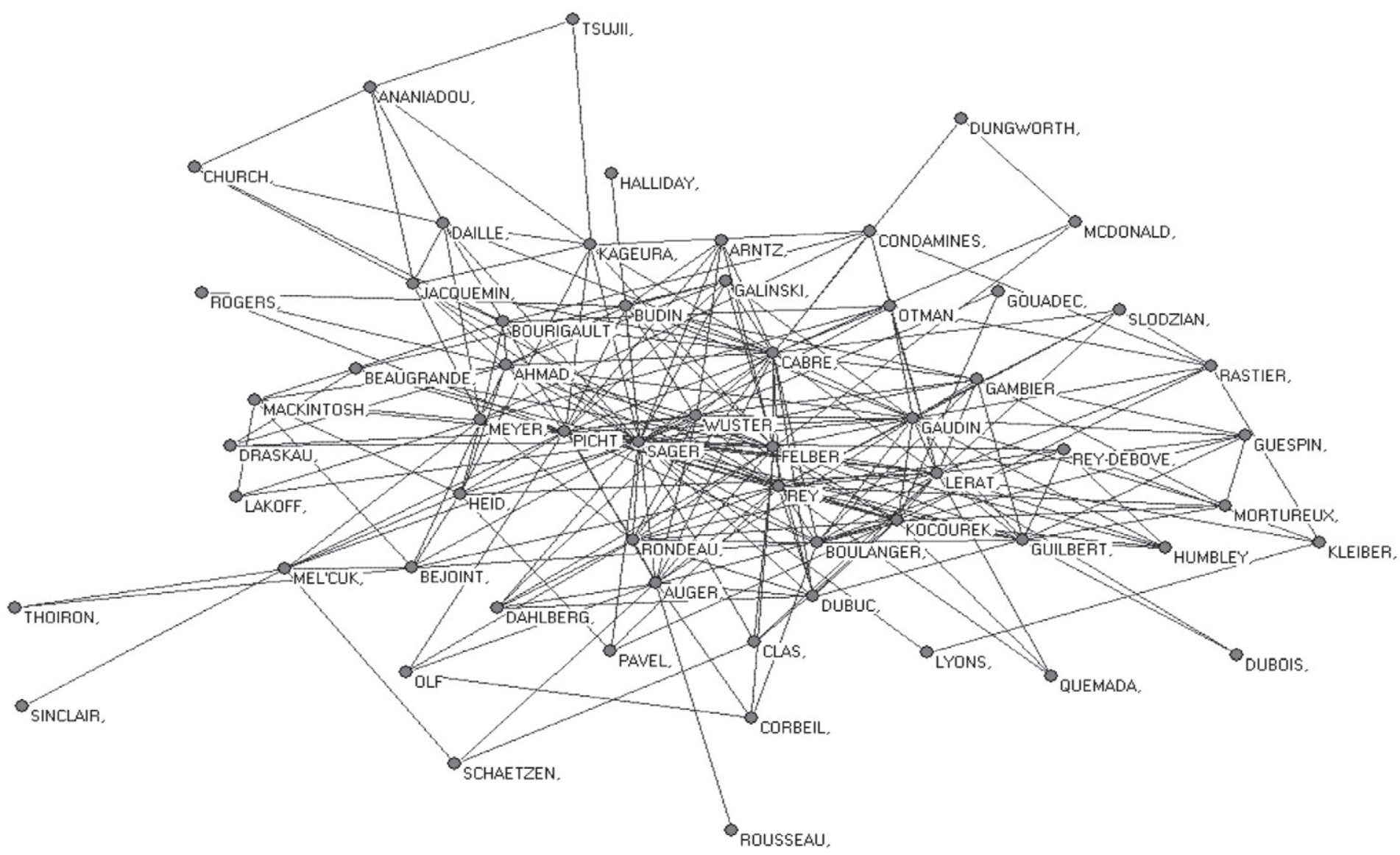


GRÁFICO 2

Representación de los principales elementos intermediarios de la red

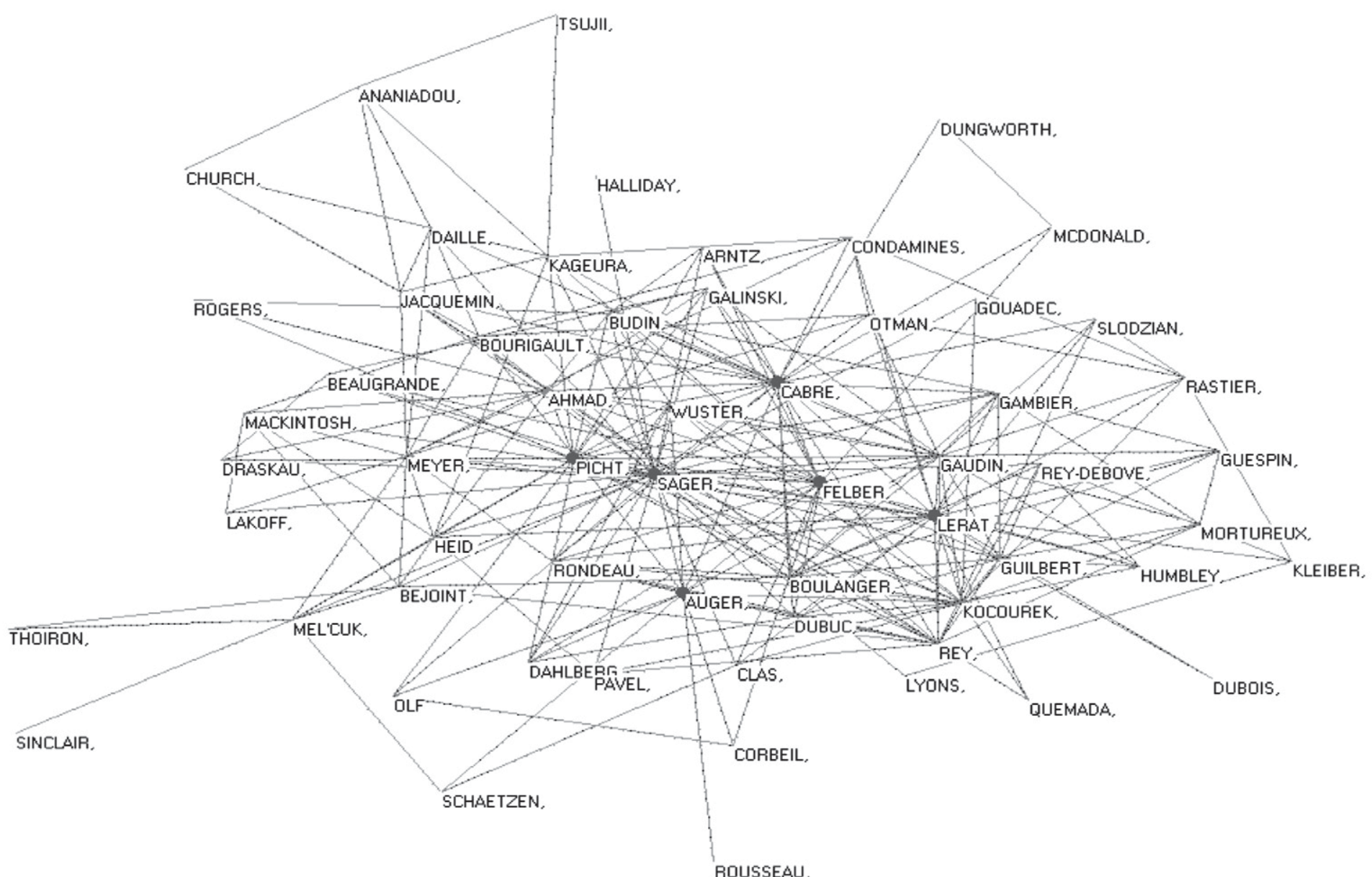


GRÁFICO 3

Representación de los vínculos más intensos de la red

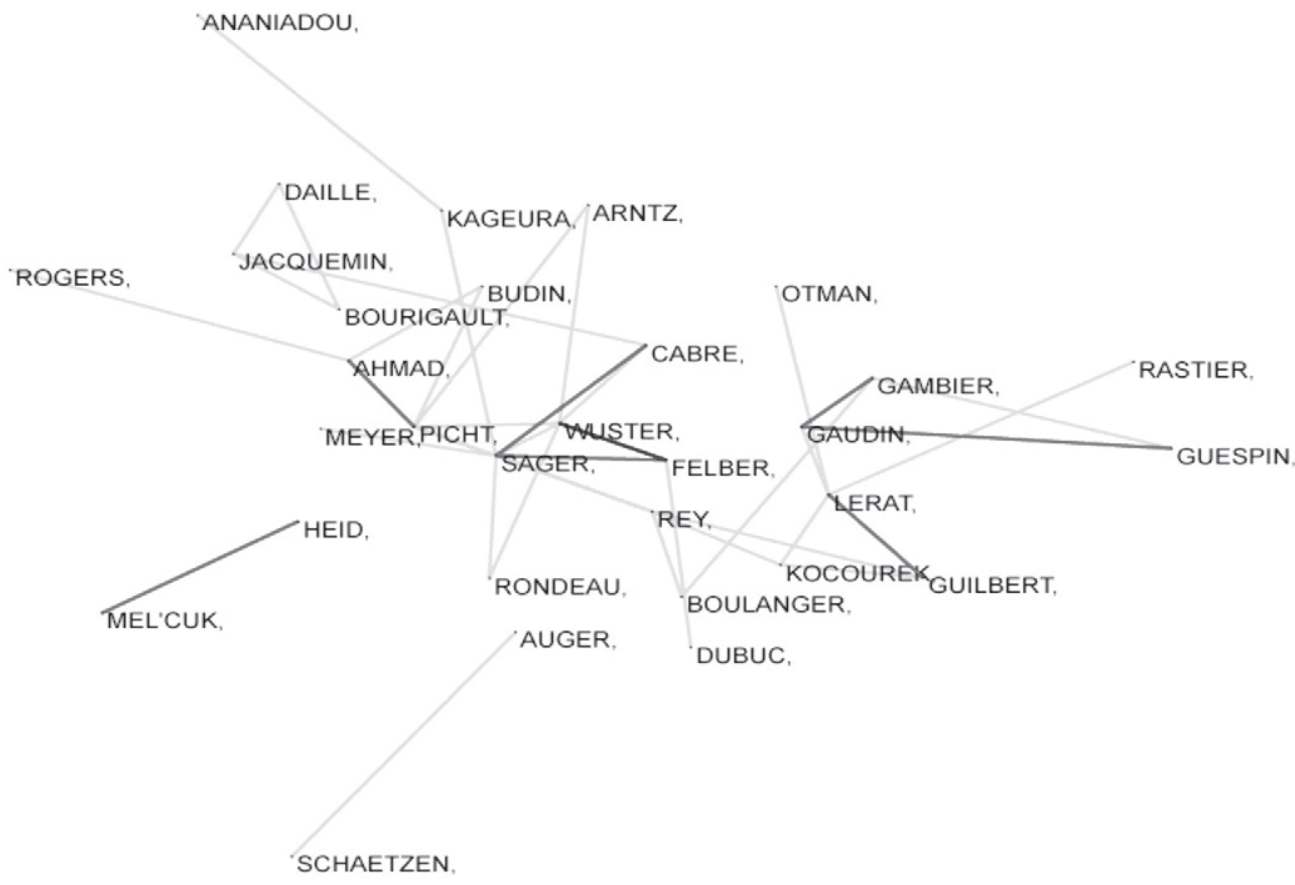

\section{Conclusiones}

La presente investigación representa la primera aproximación de la aplicación de la metodología del ACA al ámbito de la terminología, lo que ha permitido demostrar con datos objetivos el desarrollo de la disciplina estudiada. De hecho, la coincidencia elevada de los resultados de las técnicas aplicadas con la observación de la realidad de la materia estudiada demuestra la validez del método empleado.

Ya se ha reiterado que el análisis de la evolución de una disciplina se realiza gracias a ciertos datos recogidos de las revistas científicas, pues este tipo de publicaciones están consideradas como una vía rápida y eficaz de difusión del conocimiento. Para establecer el panorama científico global de la terminología, se ha tomado como punto de partida de la investigación la descripción de las publicaciones periódicas que la conforman. En consecuencia, las revistas -los artículos que contienen y los temas que tratan-, así como los autores -como productores y como referentes- han permitido establecer un acercamiento inicial a la materia desde la óptica de la producción científica.

Por todo ello, puede concluirse que, en el caso de las revistas, debido al carácter transdisciplinar de la materia, la producción en terminología, en mayor o menor medida, está dispersa en revistas pertenecientes a diversos ámbitos del conocimiento. Aunque las revistas que conforman la fuente de datos de este estudio responden temáticamente a un mismo patrón, los datos expuestos muestran una gran discontinuidad en la producción científica en las revistas de terminología, si bien se observa 
un importante punto de inflexión hacia mediados de los años noventa, propiciado por un incremento del número de los trabajos publicados que, decisivamente, repercute en el aumento de las citas incluidas en los trabajos. Este aumento está en consonancia con un cambio en los hábitos y en la concepción de estas publicaciones, tal y como ocurre en otras muchas áreas.

Como se ha tenido ocasión de comprobar, con relación a los autores, estos pueden ser estudiados en su vertiente productiva, esto es, como autores de artículos, y en su vertiente relativa al consumo de la información, esto es, como autores citados.

En el primer caso, o sea, como productores, destaca el hecho de que solo un $12 \%$ de los autores tienen una presencia regular en las publicaciones periódicas o, lo que es lo mismo, son autores de más de tres artículos. En el segundo caso, o sea, como autores citados, solo un $1 \%$ del total tiene un volumen elevado de citas. Se ha tomado este porcentaje de autores -debido a su alta presencia en las publicaciones periódicas, constituyen el grupo que arrojan resultados relevantes- como base para poder efectuar un análisis intelectual de la terminología.

La aplicación de los procedimientos dentro del marco del análisis de cocitas, que pone a nuestro alcance el método de análisis de las redes sociales, nos proporciona una "foto fija" del estado de la disciplina representada mediante sus autores más destacados, que, a su vez, simbolizan sus frentes de investigación, pues la idea fundamental sobre la que gravita este método empírico es la de la transferencia del conocimiento científico.

El ACA, por tanto, se transforma en una valiosa herramienta que nos permite estudiar el panorama investigador de la terminología, además de representar el consenso intelectual del colectivo de autores que conforman la disciplina y de exponer las relaciones sociales a través de las redes de comunicación y de conocimiento que generan sus autores. Esta fotografía fija coincide plenamente con el conocimiento interiorizado que poseen los expertos de esta materia y, a la vez, sirve de base para que el neófito se aproxime y adquiera ese conocimiento, además de permitir que el experto conozca los aspectos más descuidados, o menos estudiados, de la materia y pueda advertir las tendencias más arraigadas o las más productivas. Sin embargo, también es posible analizar la evolución de una disciplina generando mapas para periodos de tiempo sucesivos. No obstante, en el caso de la terminología la escasa producción bibliográfica de la disciplina impide un estudio diacrónico. Quizá en un futuro no muy lejano pueda llevarse a cabo.

Los resultados que ha arrojado la aplicación de esta metodología a los datos coinciden con los proporcionados por el análisis multivariable (Castro-Prieto 2007). Pero, además de ofrecer conjuntos de autores vinculados según frentes de investigación, esta técnica también indica, mediante la aplicación de una serie de elementos, la prominencia de un grupo de autores frente al resto. En efecto, Cabré, Felber, Picht y Sager indican un mayor grado de centralidad en la red y, como consecuencia de ello, se puede afirmar que son los que concentran un mayor número de conexiones con los restantes autores, además de garantizar, en el menor tiempo posible, la circulación de la información.

De las agrupaciones que se han obtenido, se perfilan nítidamente tres de ellas en todas las soluciones aportadas de la aplicación de las técnicas, independientemente del método en el que se integran. Como consecuencia, se puede afirmar que son tres las especialidades mayoritarias en la disciplina: fundamentos teóricos, pro- 
cesamiento del lenguaje natural y socioterminología. No afirmamos aquí que éstas sean las escuelas o corrientes de pensamiento existentes en esta disciplina sino que constituyen los tres frentes de investigación con los que los autores han estado más identificados durante el período 1967-2001. Para delimitar escuelas dentro de la disciplina, varias circunstancias, que en la materia no se dan en la actualidad, deberían entrar en juego. Principalmente, nos referimos al factor cronológico, ya que es necesario adquirir una cierta perspectiva temporal para dar lugar a que el pensamiento científico evolucione y se perfile adecuadamente. Por otra parte, el factor de la productividad científica se revela como fundamental para que el número, no solo de citas, sino también de autores citados, aumente. De esta forma el cruce del conocimiento será mayor, lo que permitirá una mayor precisión en las agrupaciones de autores.

Solla Price (1963), en su destacado trabajo Little Science, Big Science, afirma que la investigación científica parece seguir una curva en forma de $S$-denominada curva logística-. En efecto, en el comienzo de la curva es posible indicar una primera etapa de aparición del paradigma; su continuación representa una segunda etapa de multiplicación de las contribuciones, asociada a la aparición de los denominados colegios invisibles, es decir, círculos de investigadores influidos por unos pocos autores de alta productividad; se pasa entonces a una tercera fase de madurez; y, en la parte final de la segunda curva, a una última etapa de estancamiento. Los resultados derivados del análisis de los datos nos muestran un panorama poco consolidado de la terminología, a pesar de que su origen como materia se remonta al primer tercio del siglo pasado. Por lo tanto, continuando en la línea de Solla Price, se puede afirmar que la terminología es una materia todavía joven, pues está en su segundo estadio, en la que predominan un número reducido de autores con una gran productividad, tal y como se desprende de los resultados obtenidos en el epígrafe cuarto, por lo que aún le queda bastante camino que recorrer para llegar a su estado de madurez.

\section{REFERENCES}

Batagelj, V. and A. Mrvar (1996): "Networks/Pajek. Program for large network analysis", Página web, [consulta 14 2003], <http://vlado.fmf.uni-lj.si/pub/networks/pajek>.

Braam, R. R, Moed, H. F, and A. F. J. van Raan(1991): "Mapping of science by combined cocitation and word analysis, I: structural aspects and II: dynamical aspects", Journal of the American Society for Information Science, 42, pp. 233-266.

Castro-Prieto, M. R. (2007): Análisis de la terminología a través de la producción científica. Estudio experimental de la disciplina, Tesis doctoral, Dirigida por Pamela Faber Benítez, María Dolores Olvera Lobo, Granada, Universidad de Granada, <http://adrastea.ugr.es/ search ${ }^{\star}$ spi $\sim 4 />$.

Castro-Prieto, M. R. y M. D. Olvera-Lobo (2003a): “Aproximación a la disciplina terminológica a través de las revistas especializadas", I Congreso Internacional de la Asociación Ibérica de Estudios de Traducción e Interpretación (AIETI), pp. 515-524.

Castro-Prieto, M. R. y M.D. Olvera-Lobo (2003b): "Producción científica de la terminología: aproximación mediante el análisis de citas de las revistas especializadas”, en Terminología y traducción: un bosquejo de su evolución, pp. 159-170.

Chen, C. (1999): "Visualizing semantic spaces and author co-citation networks in digital libraries", Information Processing and Management 35-2, pp. 401-420.

Chen, C., Paul, R. J. and B. O’Keefe (2001): "Fitting the jigsaw of citation: Information visualization in domain Analysis". Journal of the American Society for Information Science and Technology, 52-4, pp. 315-330. 
Ding, Y. G., Chodbury, G. and S. Foo (2001): "Bibliometric cartography of information retrieval research by using co-word analysis". Information Processing and Management 37, pp. 817842 [consulta 22 2001], <http://dois.mimas.ac.uk/DoIS/data/Articles/juljuljiqy:2001:v:37: i:6:p:817-842.html>.

Еом, S. B. (1996): "Mapping the intellectual structure of research in decision support systems through author co-citation analysis", Decision Support Systems, 16, pp. 315-318.

Freeman, L. C. (2000): "La centralidad en las redes sociales. Clarificación conceptual", Política y Sociedad. Monográfico "Análisis de Redes Sociales: La Consolidación de un Paradigma Interdisciplinar", 33, pp. 131-148.

Garfield, E. (1994): "The concept of Citation Indexing: A unique and innovative tool for navigating the research literature", Currents Contents, 3, pp. 3-5.

González de Dios, Moya, J. M. y M. A. Mateos (1997): “Indicadores bibliométricos: características y limitaciones en el análisis de la actividad científica", Anales Españoles de Pediatría, 47-3, pp. 235-244.

LEydersdorfF, L. (1998): “Theories of citation?”, Scientometrics, 43-1, pp. 5-25.

Marshakova, V. (1973): "System of document connections based on references", NauchnoTekhnichescaya Informatisya: Series II 6, pp. 3-8.

McCAIn, K. W. (1990): "Mapping authors in intellectual space: A technical overview". Journal of the American Society for Information Science, 41-6, pp. 433-443.

SMALl, H. (1973): "Co-citation in scientific literature: a new measure of the relationship between publications". Journal of the American Society for Information Science, 24-4, pp. 265-269.

Small, H.(1997): "Update on science mapping: creating large document spaces", Scientometrics, 38-2, pp. 275-293.

Small, H. (1998): "A general framework for creating large scale maps of science in two or three dimensions: the SciViz system". Scientometrics, 41-1/2, pp. 125-133.

Small, H. and B. C. Griffith (1974): "The structure of scientific literatures. I: identifying and graphing specialities”, Science Studies 4, pp. 17-40.

Small, H., Sweeney, E. and E. Greenlee (1985): "Clustering the science citation index using co-citations. II. Mapping science”, Scientometrics, 8-5/6, pp. 321-340.

Solla Price, D. J. (1963): Little Science, Big Science, New York, Columbia University Press.

Wasserman, S. y K. Faust (1994): Social Network Analysis. Methods and Applications. Structural Analysis in the Social Sciences, Cambridge, United Kingdom, Cambridge University Press.

White, H. D. (1986): "Cocited author retrieval", Information Technology and Libraries, 5-2, pp. 93-99.

White, H. D. and B.C. Griffith (1981): "Author co-citation: A literature measure of intellectual structure", Journal of the American Society for Information Science, 32, pp. 163-172.

White, H. D. and B.C. Griffith (1982): "Authors as markers of intellectual space: co-citation in studies of science, technology and society", Journal of Documentation, 38, pp. 255-272.

White, H. D. and K.W. McCain (1998): "Visualizing a Discipline: An author Co-citation Analysis of Information Science, 1972-1995", Journal of the American Society for Information Science, 49-4, pp. 327-355. 